\title{
Alamouti-coded decode-and-forward protocol with optimum relay selection and power allocation for cooperative communications
}

\author{
Wanakorn Swasdio ${ }^{1}$, Chaiyod Pirak ${ }^{1 *}$, Somchai Jitapunkul ${ }^{2}$ and Gerd Ascheid ${ }^{3}$
}

\begin{abstract}
In this paper, an Alamouti-coded decode-and-forward protocol with optimum relay selection for three-user one destination cooperative communications is investigated. Particularly, we design the new protocol that allows the source node retransmitting the signal to the destination node at the same time as the relay node forwarding the received signal to the destination node with an Alamouti coding scheme. We exploit the cooperative maximum ratio combining technique (C-MRC) at the destination for combining a multiple copy of received signals. Therefore, the proposed scheme achieves the maximum diversity gain and lower probability of error in comparison with the existing decode and forward protocol. We also analyze a symbol error rate (SER) upper bound of the single relay system, and exploit a result of this analysis to select the optimum relay in a multiple-relay cooperation scheme based on the minimum SER selection strategy. Moreover, the optimum power allocation for the proposed protocol is derived, and it is able to provide the optimum transmission power strategy to the source and the relay nodes in order to achieve the minimum probability of error. In the performance analysis, the theoretical error probability is studied and compared with the simulation results. Simulation results indicate that the proposed protocol significantly outperforms the existing protocols, and the theoretical error probability curve is relatively close to the simulated SER curve.
\end{abstract}

Keywords: Cooperative communications; Alamouti coding; Optimum relay selection; Optimum power allocation

\section{Introduction}

In the future multimedia communications, broadband wireless communications will play a major role in personal voice and data communications that support fixed, nomadic, portable, and mobile accesses. There are many techniques that could be able to enhance the performance of wireless communications, including a multiple-input multiple-output (MIMO) system. However, given the current technology, equipping more antennas to handheld devices is far from practical. Recently, a generalized MIMO system, called cooperative communications [1], has been proposed for realizing the advantage of the conventional MIMO system, e.g., a diversity gain. By the cooperation of active users equipped with a single antenna in the wireless

\footnotetext{
* Correspondence: chaiyod.p.ce@tggs-bangkok.org

${ }^{1}$ The Sirindhorn International Thai-German Graduate School of Engineering (TGGS), King Mongkut's University of Technology North Bangkok, Bangkok 10800, Thailand

Full list of author information is available at the end of the article
}

network, cooperative communications can be established in a distributed fashion.

It is well-known that Alamouti's space-time coding [2] could enhance the performance of wireless communication systems over flat fading channels by the virtue of the diversity gain obtained from the exploitation of two antennas at the transmitter. Furthermore, the decoding algorithm is also practically feasible with an acceptable complexity. In [3], a probability of error performance analysis for decode-and-forward cooperation protocol in wireless networks has been proposed, where the comparison between a closed-form symbol error rate (SER) formulation and an upper bound approximation have been investigated. In [4], the new combining technique, namely a cooperative maximum ratio combining (C-MRC), is proposed to overcome the diversity gain limitation of the conventional decode-and-forward protocol. In [5], the Hurwitz-Radon space-time code for the wireless relay network has been proposed. The results show that 
the diversity factor depends on the number of relays, and a 10-dB signal-to-noise ratio (SNR) improvement compared with the single relay system is achieved. In [6], the authors show the closed-form expression of the average symbol error probability for a distributed Alamouti scheme in wireless relay networks and examine such scheme by using the Monte Carlo simulation. In [7], the authors investigate a difference between the diversity of multiple relays and the diversity of multiple receivers/ transmitters, and show the switching scheme with the space-time modulation that is able to reduce a bit error rate in the wireless relay communications. In [8], the performance analysis of Alamouti's coded-based cooperative communication has been proposed, where the system uses an amplify-and-forward (AF) strategy over Nakagami's fading. In addition, the system performance is not fully optimized by using this strategy because of the problem of synchronization. The best-relay selection scheme for cooperative networks has been introduced in [9], and it is called an opportunistic relaying. According to the opportunistic relaying, a single relay among a set of relay nodes is selected, depending on which relay provides the 'best' end-to-end path between source and destination nodes. The authors in [9] show that this scheme yields the same diversity order as the cooperative communication using a space-time coding in both decode-and-forward (DF) and amplify-and-forward protocols. The authors in [10] investigate the performance of the best-relay selection scheme, where the 'best' relay only participates in the relaying phase. Therefore, two channels are only needed in this case (one for the direct link and another one for the best relay link) regardless of the number of relays. The best relay is defined as the relay node that is able to yield the highest signal-to-noise ratio at the destination node. In [11], a two-hop cooperative multiple-relay communication network is considered. The authors propose the exact outage and capacity performance expression for relay selection over a wide range of SNR regimes. In [12], the authors propose a new relay selection scheme for cooperative decode-and-forward with multiple antennas that achieve a full diversity order.

Referring to the existing Alamouti-based decode-andforward protocol in [3], it is obvious that the degree-offreedom of the system, i.e., a possible opportunity of signal transmission in all available time slots, is not fully used. Furthermore, such system performance could be enhanced by the cooperative maximum ratio combining (C-MRC) technique, and the bandwidth efficiency could also be increased by using the optimum relay selection technique. These facts motivate us to propose the new protocol, and the main contributions of this paper are as follows.

In this paper, we propose the Alamouti-coded decodeand-forward protocol with an optimum relay selection technique for cooperative communications. The system allows the source node transmitting the signal in the second time slot at the same time as the relay node is decoding and forwarding the signal that is received from the source node in the first time slot to the destination using Alamouti's coding scheme. At the destination node, the received signal from both source and relay nodes will be combined by using a cooperative maximum ratio combining technique. In the C-MRC technique, the received signal sent by the relay node at the destination is weighted by the quality of the channel, i.e., a ratio of channel variances, before combining with the received signal sent by the source node resulting in an enhanced error probability and signal-to-noise ratio (SNR). The proposed scheme provides a much lower error probability in comparison with existing cooperative protocols. We also analyze a symbol error rate upper-bound for the Alamouti-coded decode-and-forward protocol with the C-MRC signal combining, and evaluate the analysis results by comparing with the simulation results. Based on this analysis, we can select the optimum relay that provides the lower probability of error for cooperative communications. Finally, we derive the optimum power allocation for the proposed system by minimizing the upper-bound on probability of error, and we are able to provide the optimum power transmission strategy to the source and relay nodes for data transmission. In the performance analysis section, the result shows that the theoretical error probability curve is close to that the simulated one, and the error probability of the optimum relay selection strategy is lower than the fixed relay strategy.

The rest of this paper is organized as follows: In section 2, we describe the system and received signal models. In section 3, we analyze the total SNR of the system and the expressions of MRC and C-MRC signal combining techniques for Alamouti-coded decode-and-forward protocol. In section 4, we analyze the average SER of the proposed system and propose the optimum relay selection technique for the Alamouti-coded decode-and-forward protocol. We also investigate the optimum power allocation for the proposed system in this section. In section 5, the simulation results are shown, and we conclude this paper in section 6.

\section{System and received signal models 2.1 System model}

In this section, we consider the Alamouti-coded decodeand-forward protocol with relay selection for cooperative communications. We consider three users and one destination (D) in the system, in which the first user acts as a source ( $\mathrm{S}$ ) and other users act as relays (R), as shown in Figure 1.

In Figure 1, we assume that each relay knows its sourceto-relay and relay-to-destination channel variances. Next, each relay sends this channel information to the source 


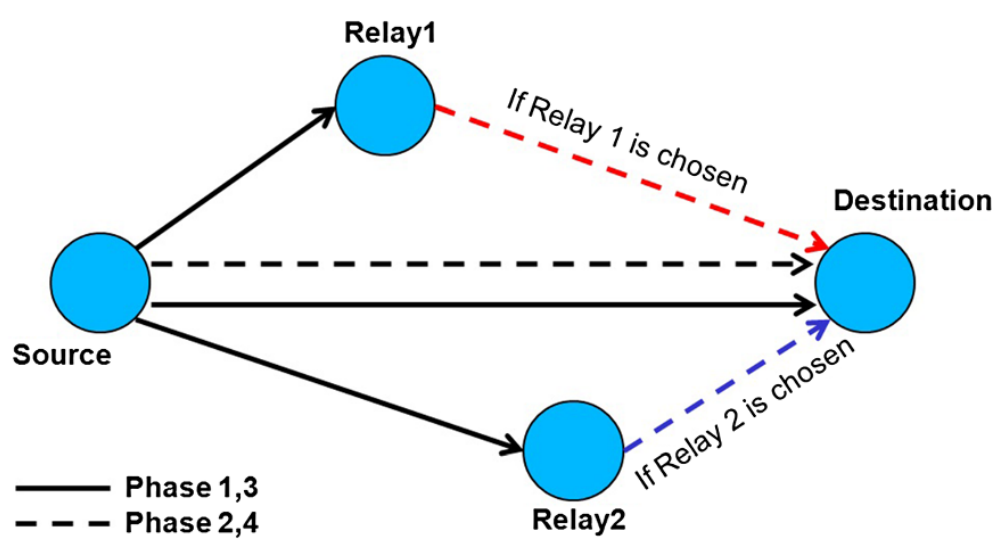

Figure 1 The system model of an Alamouti-coded decoded-and-forward protocol with relay selection for cooperative communications.

node through some feedback channels. Thus, the source can compute and select only one optimum relay to cooperate with by the decision algorithm with the feedback information. We employ a decode-and-forward protocol in the broadcasting phase, in which the best relay node receives the source's transmitted signal, decodes and re-modulates it, and retransmits this newly modulated signal to the destination, as shown in Figure 2. In the relaying phase, the transmitting symbols from the source and relay nodes will be encoded by Alamouti's space-time coding, as shown in the following matrix [2]:

$$
C=\left(\begin{array}{cc}
S_{1} \stackrel{\text { time }}{\rightarrow} & S_{2} \\
-S_{2}^{*} & S_{1}^{*}
\end{array}\right) \downarrow_{\text {space }}
$$

where $C$ is the Alamouti's coding matrix, and $S_{1}$ and $S_{2}$ are the transmitting symbols in two consecutive time slots.

In Figure 2a,b, it is worth noticing that we also exploit the source-to-destination symbols in the broadcasting phases, i.e., phases 1 and 3, as additional received signals for combining with the source-to-destination symbols in the relaying phases, i.e. phases 2 and 4 , by using the cooperative maximum ratio combining technique in order to enhance the SNR of the received signal sent by the source node. Therefore, the error probability will be correspondingly reduced. In addition, this benefit is achieved by a full utilization of signal transmission in both broadcasting and relaying phases of cooperative communications. By integrating the proposed cooperative communications with the Alamouti's coding scheme, we could achieve a full diversity gain with the enhanced SNR for the source-to-destination received signal. We divide our communications into four phases as follows.

In phase 1 of Figure 2a, the source node broadcasts its modulated signal to the selected relay (based on the optimum relay selection technique described in section 4.2) and the destination nodes through wireless channels. In phase 2 of Figure 2a, the relay node decodes and forwards the received signal, sent by the source node, to the destination node. In this relaying phase, the source node transmits another signal to the destination node based on the Alamouti's coding. Similarly, the signal transmission in phases 3 and 4 in Figure 2b is equivalent to phases 1 and 2 , respectively, except the symbol encoding has to obey the Alamouti's coding matrix, as shown in (1). We employ a time-division multiple access (TDMA) scheme for signal transmission, and we consider a half-rate communication with the QPSK modulation so that the bandwidth efficiency equals $1 \mathrm{bit} / \mathrm{s} / \mathrm{Hz}$. Essentially, we will lose four times in one communication frame to transmit two symbols so that we employ the QPSK modulation to compensate such bandwidth efficiency loss.

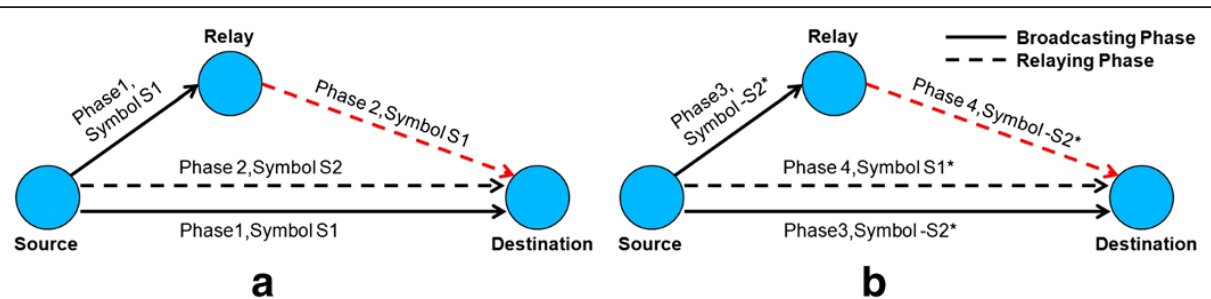

Figure 2 Proposed Alamouti-coded decoded-and-forward protocol for cooperative communications in different phases. (a) Phases 1 and 2 and (b) phases 3 and 4 . 


\subsection{Received signal model}

We divide the received signal into four phases. In phase 1 , the received signals at the destination and relay nodes sent by the source node are described in (2) and (3), respectively. In phase 2 , the received signal at the destination node, that is simultaneously sent by relay and source nodes based on the proposed protocol shown in Table 1, is described in (4).

Phase 1:

$$
\begin{aligned}
& y_{d 1}=\sqrt{P_{1}} H_{d 1} S_{1}+n_{d 1} \\
& y_{r 1}=\sqrt{P_{1}} H_{r 1} S_{1}+n_{r 1}
\end{aligned}
$$

Phase 2:

$$
y_{d 2}=\sqrt{w P_{1}} H_{d 2} S_{2}+\sqrt{w P_{2}} H_{r 2} \hat{S}_{1}+n_{d 2}
$$

where $y_{d 1}$ and $y_{r 1}$ are the received signals at the destination and the relay nodes in phase 1 , respectively, in which $S_{1}$ denotes a transmitted symbol of the source node, $y_{d 2}$ is the received signal at the destination node in phase 2 , in which the source node transmits a symbol $S_{2}$ and the relay node decodes and forwards the decoded symbol $\hat{S}_{1} ; P_{1}$ and $P_{2}$ are the transmit power of the source and the relay nodes, respectively; $H_{d 1}, H_{r 1}, H_{d 2}$, and $H_{r 2}$ are the channel impulse responses of the source-to-destination link in phase 1 , the source-to-relay link in phase 1 , the source-todestination link in phase 2 , and the relay-to-destination link in phase 2 , respectively. In addition, we introduce a weighting factor $w$ in phase 2 based on a concept of C-MRC signal combining, $y_{d 1}$ in which $w=\gamma_{\mathrm{eq}} / \gamma_{\mathrm{rd}}$, where $\gamma_{\mathrm{eq}}=\min \left(\gamma_{\mathrm{sv}}, \gamma_{\mathrm{rd}}\right)$, and $\gamma_{\mathrm{sr}}$ and $\gamma_{\mathrm{rd}}$ are the instantaneous SNR between the source-to-relay link and the relayto-destination link, respectively. Furthermore, $n_{d 1}, n_{r 1}$, and $n_{\mathrm{d} 2}$ are zero-mean complex additive white Gaussian noise (AWGN) with variance $N_{0}$ at the destination in phase 1 , at the relay in phase 2 , and at the destination nodes in phase 2, respectively. Basically, the Alamouti's coding scheme uses two time slots to transmit two symbols. Hence, the received signals of phases 3 and 4 can be described as follows:

Phase 3:

$$
\begin{aligned}
& y_{d 3}=\sqrt{P_{1}} H_{d 3}\left(-S_{2}^{*}\right)+n_{d 3} \\
& y_{r 3}=\sqrt{P_{1}} H_{r 3}\left(-S_{2}^{*}\right)+n_{r 3}
\end{aligned}
$$

Phase 4:

$$
y_{d 4}=\sqrt{w P_{1}} H_{d 4} S_{1}^{*}+\sqrt{w P_{2}} H_{r 4}\left(-\hat{S}_{2} *\right)+n_{d 4}
$$

where $y_{d 3}$ and $y_{r 3}$ are the received signals at the destination and the relay nodes in phase 3 , respectively, $y_{d 4}$ is the received signal at the destination node in phase 4 , in which the source node transmits a symbol $S_{1}^{*}$ and the relay node decodes and forwards the decoded symbol $-S_{2}^{*}$. In addition, $n_{d 3}, n_{r 3}$, and $n_{\mathrm{d} 4}$ are zero-mean complex additive white Gaussian noise (AWGN) with variance $N_{0}$ at the destination in phase 3 , at the relay in phase 4 , and at the destination nodes in phase 4 , respectively. We now assume that all channels are modeled as quasi-static Rayleigh flat-fading channels, i.e., $H_{d 1}=H_{d 2}=H_{d 3}=H_{d 4}$, $H_{r 1}=H_{r 3}$ and $H_{r 2}=H_{r 4}$, within one transmission frame according to a typical assumption for the Alamouti's coding scheme [2].

\section{Signal combining technique}

In this section, we combine the received signals in all phases in order to detect $S_{1}$ and $S_{2}$ by using a maximum ratio combining (MRC) technique and a cooperative maximum ratio combining (C-MRC) technique. We also derive a closed-form expression of the total SNR resulted from such combining techniques. It could be shown that the output of the MRC detector at the destination can be expressed as follows:

$$
\gamma_{\text {total }}=\gamma_{\text {direct }}+\gamma_{\text {Alamouti }}
$$

where $\gamma_{\text {total }}$ is the total SNR, $\gamma_{\text {direct }}$ is the SNR of a symbol in phase 1 or 3 , and $\gamma_{\text {Alamouti }}$ is the SNR of a symbol in phase 2 or 4 .

Table 1 Alamouti-coded decoded-and-forward protocol with relay selection for cooperative communication

\begin{tabular}{lll}
\hline Timing & Tx & Rx \\
\hline Phase 1 (Direct) & S: Broadcast $S_{1}$ to $R$ and $D$ & R: Receive $S_{1}$ from $S$ \\
& D: Receive $S_{1}$ from $S$ & D: Receive $S_{1}$ from $R$, and $S_{2}$ from $S$ with Alamouti's coding \\
Phase 2 (Alamouti) & R: Retransmit $S_{1}$ to $D$ & D: Receive $-S_{2}^{*}$ from $S$ \\
Phase 3 (Direct) & $S:$ Broadcast $-S_{2}^{*}$ to $R$ and $D$ & R: Receive $-S_{2}^{*}$ from $S$ \\
Phase 4 (Alamouti) & $S:$ Transmit $S_{1}^{*}$ to $D$ & D: Receive $-S_{2}^{*}$ from $R$, and $S_{1}^{*}$ from $S$ with Alamouti's coding \\
& $R:$ Retransmit $-S_{2}^{*}$ to $D$ & \\
\hline
\end{tabular}




\subsection{Maximum ratio combining (MRC) technique}

The maximum ratio signal combining for the received signals in (2), (4), (5), and (7) can be expressed as [13]

$$
\begin{aligned}
y_{S_{2}^{*}(\mathrm{MRC})}= & w_{21(\mathrm{MRC})}\left(-y_{d 3}^{*}\right)+w_{22(\mathrm{MRC})} y_{d 2} \\
& +w_{23(\mathrm{MRC})}\left(-y_{d 4}^{*}\right) \\
y_{S_{1}^{*}(\mathrm{MRC})}= & w_{11(\mathrm{MRC})} y_{d 1}+w_{12(\mathrm{MRC})} y_{d 2}+w_{13(\mathrm{MRC})} y_{d 4}^{*}
\end{aligned}
$$

where $y_{S_{1}^{\prime}(\mathrm{MRC})}$ and $y_{S_{2}(\mathrm{MRC})}$ are the output of the MRC combiner at the destination, and

$$
\begin{aligned}
& w_{11(\mathrm{MRC})}=\sqrt{P_{1}} H_{d 1}^{*} / N_{0}, w_{21(\mathrm{MRC})}=\sqrt{P_{1}} H_{d 3} / N_{0} \\
& w_{12(\mathrm{MRC})}=\sqrt{P_{2}} H_{r 2}^{*} / N_{0}, w_{22(\mathrm{MRC})}=\sqrt{P_{1}} H_{d 2}^{*} / N_{0} \\
& w_{13(\mathrm{MRC})}=\sqrt{P_{1}} H_{d 4} / N_{0}, w_{23(\mathrm{MRC})}=\sqrt{P_{2}} H_{r 4} / N_{0}
\end{aligned}
$$

denote the MRC weight, where $N_{0}$ is a variance of AWGN. By substituting (2), (4), (5), and (7) into (9) and (10), we have

$$
\begin{aligned}
y_{S_{1}(\mathrm{MRC})}= & \frac{P_{1}\left|H_{d 1}\right|^{2} S_{1}}{N_{0}}+\frac{P_{1}\left|H_{d 4}\right|^{2} S_{1}}{N_{0}}+\frac{P_{2}\left|H_{r 2}\right|^{2} S_{1}}{N_{0}} \\
& +N_{S 1(\mathrm{MRC})} \\
y_{S_{2}(\mathrm{MRC})}= & \frac{P_{1}\left|H_{d 3}\right|^{2} S_{2}}{N_{0}}+\frac{P_{1}\left|H_{d 2}\right|^{2} S_{2}}{N_{0}}+\frac{P_{2}\left|H_{r 4}\right|^{2} S_{2}}{N_{0}} \\
& +N_{S 2(\mathrm{MRC})}
\end{aligned}
$$

where $N_{S 1 \text { (MRC) }}$ and $N_{S 2 \text { (MRC) }}$ are the total noise at the destination node expressed by

$$
\begin{aligned}
& N_{S 2 \text { (MRC) }}=\frac{\sqrt{P_{1}} H_{d 3} n_{d 3}}{N_{0}}+\frac{\sqrt{P_{1}} H_{d 2}^{*} n_{d 2}}{N_{0}}+\frac{\sqrt{P_{2}} H_{r 4} n_{d 4}}{N_{0}} \\
& N_{S 2(\mathrm{MRC})}=\frac{\sqrt{P_{1}} H_{d 3} n_{d 3}}{N_{0}}+\frac{\sqrt{P_{1}} H_{d 2}^{*} n_{d 2}}{N_{0}}+\frac{\sqrt{P_{2}} H_{r 4} n_{d 4}}{N_{0}}
\end{aligned}
$$

For the sake of simplicity, we regard that $H_{d 1}=H_{d 2}=$ $H_{d 3}=H_{d 4}=H_{\text {direct }}$ and $H_{r 2}=H_{r 4}=H_{\text {relay }}$. The total SNR of the MRC output can be derived as follows:

$$
\begin{aligned}
& \gamma_{S 1}=\frac{2 P_{1}\left|H_{\text {direct }}\right|^{2}+P_{2}\left|H_{\text {relay }}\right|^{2}}{N_{0}} \\
& \gamma_{S 2}=\frac{2 P_{1}\left|H_{\text {direct }}\right|^{2}+P_{2}\left|H_{\text {relay }}\right|^{2}}{N_{0}}
\end{aligned}
$$

where $\gamma_{S 1}$ is the total SNR of a symbol $S_{1}$ and $\gamma_{s 2}$ is the total SNR of a symbol $S_{2}$.
It is worth noticing that the MRC combining yields the maximum SNR to (15) and (16), given that the estimated signals $\hat{S}_{1}$ and $\hat{S}_{2}$ at the relay node are correctly decoded. Specifically, in practical applications, the correctness of symbols $\hat{S}_{1}$ and $\hat{S}_{2}$ solely depends on the quality of the channel link from the source to relay link. Hence, the MRC combining cannot guarantee the maximum SNR as mentioned in [9].

\subsection{Cooperative maximum ratio combining technique}

The cooperative maximum ratio signal combining for the received signals in (2), (4), (5), and (7) could be expressed as follows [4]:

$$
\begin{aligned}
y_{S_{1}^{*}(\mathrm{CMRC})}= & w_{11(\mathrm{CMRC})} y_{d 1}+w_{12(\mathrm{CMRC})} y_{d 2} \\
& +w_{13(\mathrm{CMRC})} y_{d 4}^{*} \\
y_{S_{2}^{*}(\mathrm{CMRC})}= & w_{21(\mathrm{CMRC})}\left(-y_{d 3}^{*}\right)+w_{22(\mathrm{CMRC})} y_{d 2} \\
& +w_{23(\mathrm{CMRC})}\left(-y_{d 4}^{*}\right)
\end{aligned}
$$

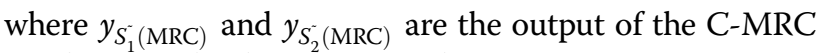
combiner at the destination and

$$
\begin{aligned}
& w_{11(\mathrm{CMRC})}=\sqrt{P_{1}} H_{d 1}^{*} / N_{0}, w_{21(\mathrm{CMRC})}=\sqrt{P_{1}} H_{d 3} / N_{0} \\
& w_{12(\mathrm{CMRC})}=\sqrt{w P_{2}} H_{r 2}^{*} / N_{0}, w_{22(\mathrm{CMRC})}=\sqrt{w P_{1}} H_{d 2}^{*} / N_{0} \\
& w_{13(\mathrm{CMRC})}=\sqrt{w P_{1}} H_{d 4} / N_{0}, w_{23(\mathrm{CMRC})}=\sqrt{w P_{2}} H_{r 4} / N_{0}
\end{aligned}
$$

denote the C-MRC weight where $N_{0}$ is a variance of AWGN. By substituting (2), (4), (5), and (7) into (17) and (18), we have

$$
\begin{aligned}
y_{S_{1}^{*}(\mathrm{CMRC})}= & \frac{P_{1}\left|H_{d 1}\right|^{2} S_{1}}{N_{0}}+\frac{w P_{1}\left|H_{d 4}\right|^{2} S_{1}}{N_{0}}+\frac{w P_{2}\left|H_{r 2}\right|^{2} S_{1}}{N_{0}} \\
& +N_{S 1(\mathrm{CMRC})} \\
y_{S_{2}(\mathrm{CMRC})=} & \frac{P_{1}\left|H_{d 3}\right|^{2} S_{2}}{N_{0}}+\frac{w P_{1}\left|H_{d 2}\right|^{2} S_{2}}{N_{0}}+\frac{w P_{2}\left|H_{r 4}\right|^{2} S_{2}}{N_{0}} \\
& +N_{S 2(\mathrm{CMRC})}
\end{aligned}
$$

where $N_{S 1 \text { (C-MRC) }}$ and $N_{S 2 \text { (C-MRC) }}$ are the total noise at the destination node

$$
\begin{aligned}
& N_{S 1(\mathrm{CMRC})}=\frac{\sqrt{P_{1}} H_{d 1}^{*} n_{d 1}}{N_{0}}+\frac{\sqrt{w P_{1}} H_{d 4} n_{d 4}}{N_{0}}+\frac{\sqrt{w P_{2}} H_{r 2}^{*} n_{d 2}}{N_{0}} \\
& N_{S 2(\mathrm{CMRC})}=\frac{\sqrt{P_{1}} H_{d 3} n_{d 3}}{N_{0}}+\frac{\sqrt{w P_{1}} H_{d 2}^{*} n_{d 2}}{N_{0}}+\frac{\sqrt{w P_{2}} H_{r 4} n_{d 4}}{N_{0}}
\end{aligned}
$$

In the concept of C-MRC, it is worth noticing that the quality of the decoded symbols $\hat{S}_{1}$ and $\hat{S}_{2}$ greatly depends 
on the SNR of the source-to-relay link. Specifically, if $\gamma_{\mathrm{sr}}<\gamma_{\text {rd }}$, i.e., more error could be anticipated on $\hat{S}_{1}$ and $\hat{S}_{2}$, then the confidence is reduced on the source-to-relay link. This fact results in the expression of $w=\gamma_{\mathrm{eq}} / \gamma_{\mathrm{rd}}$, where $\gamma_{\mathrm{eq}}=\min \left(\gamma_{\mathrm{sv}} \gamma_{\mathrm{rd}}\right)$, and $\gamma_{\mathrm{sr}}$ and $\gamma_{\mathrm{rd}}$ are the instantaneous SNR between source-to-relay and relay-to-destination links. The total SNR of the MRC output can be derived as follows:

$$
\begin{aligned}
& \gamma_{s 1}=\frac{(1+w) P_{1}\left|H_{\text {direct }}\right|^{2}+w P_{2}\left|H_{\text {relay }}\right|^{2}}{N_{0}} \\
& \gamma_{s 2}=\frac{(1+w) P_{1}\left|H_{\text {direct }}\right|^{2}+w P_{2}\left|H_{\text {relay }}\right|^{2}}{N_{0}}
\end{aligned}
$$

In this paper, we employ the C-MRC signal combining technique at the destination node because it could provide a full diversity gain, and it is superior to the MRC signal combining. Once the signal combining, i.e. (23) and (24), has been done at the destination node, the destination node's receiver will perform the symbol detection, i.e. $S_{1}$ and $S_{2}$, by using a maximum-likelihood (ML) receiver.

\section{Performance analysis}

\subsection{Probability of error analysis}

In this section, we analyze the error probability performance, i.e., a symbol error rate (SER), of the Alamouti-coded decode-and-forward protocol for cooperative communications with the C-MRC signal combining. We derive a SER upper bound for the proposed system with the M-PSK modulation. When the M-PSK modulation is used in the system, with the instantaneous SNR $(\gamma)$ in (23) and (24), the conditional SER, where the channel coefficients $H_{\mathrm{sd}}, H_{\mathrm{sr}}$ and $H_{\mathrm{rd}}$ are the channel impulse responses of the source-to-destination link, source-torelay link, and the relay-to-destination link, respectively, can be written as [14].

$$
P_{\mathrm{psk}}^{H_{\mathrm{sd}} H_{\mathrm{sr}} H_{\mathrm{rd}}} \triangleq \frac{1}{\pi} \int_{0}^{(M-1) \pi / M} \exp \left(-\frac{b_{\mathrm{psk}} \gamma_{\mathrm{total}}}{\sin ^{2} \theta}\right) d \theta
$$

where $b_{\mathrm{psk}}=\sin ^{2}(\pi / M)$ and $M=2^{k}$ with $k$ even. The channel variances of $H_{\mathrm{sd}}$ and $H_{\mathrm{rd}}$ are defined by $\delta_{\mathrm{sd}}^{2}$ and $\delta_{\mathrm{rd}}^{2}$, respectively. The conditional SER given $H_{\mathrm{sd}}, H_{\mathrm{sp}}$ and $H_{\mathrm{rd}}$ and $w$ could be expressed by

$$
P_{\mathrm{psk}}^{H_{\mathrm{sd}} H_{\mathrm{ss}} H_{\mathrm{rd} w}}=\frac{1}{\pi} \int_{0}^{(M-1) \pi / M} \exp \left(-\frac{b_{\mathrm{psk}}\left((1+w) P_{1}\left|H_{\mathrm{sd}}\right|^{2}+w P_{2}\left|H_{\mathrm{rd}}\right|^{2}\right)}{N_{0} \sin ^{2} \theta}\right) d \theta
$$

Given $|H|^{2}$ having an independent Rayleigh distribution with $\mathrm{E}\left[|H|^{2}\right]=\delta_{H}^{2}$, one can show that

$$
\int_{0}^{\infty} \exp \left(-\frac{b_{\mathrm{psk}} P_{1} z}{N_{0} \sin ^{2} \theta}\right) p_{|H|^{2}}(z) d z=\frac{1}{1+\frac{b_{\mathrm{psk}} P_{1} \delta_{H}^{2}}{N_{0} \sin ^{2} \theta}},
$$

By averaging over the Rayleigh fading channel $H_{\mathrm{sd}}, H_{\mathrm{sp}}$ and $H_{\text {rd }}$, the upper bound on conditional SER of proposed system can be expressed as

$$
P_{\mathrm{psk}}^{w} \leq F_{1}\left(1+\frac{b_{\mathrm{psk}}(1+w) P_{1} \delta_{s d}^{2}}{N_{0} \sin ^{2} \theta}\right)\left(1+\frac{b_{\mathrm{psk}} w P_{2} \delta_{\mathrm{rd}}^{2}}{N_{0} \sin ^{2} \theta}\right)
$$

where

$$
F_{1}(x(\theta))=\frac{1}{\pi} \int_{0}^{(M-1) \pi / M} \frac{1}{x(\theta)} d \theta .
$$

It is worth noticing that (28) has been derived by using the assumption that $H_{\mathrm{sd}}, H_{\mathrm{sp}}, H_{\mathrm{rd}}$, and $w$ are statistically independent; therefore, the result in (28) serves as the upper bound on conditional SER. In fact, $H_{\mathrm{sr}}, H_{\mathrm{rd}}$, and $w$ are jointly dependent.

From (28), if we substitute $\sin ^{2} \theta=1$, which is the maximum value, then all integrands reach their maximum value. The upper bound on the conditional SER of the proposed system can be expressed as $[15,16]$.

$$
P_{\mathrm{psk}}^{w} \leq \frac{(M-1)}{M} \times \frac{N_{0}^{2}}{\left(N_{0}+(1+w) A\right)\left(N_{0}+w C\right)}
$$

where $A=b_{\mathrm{psk}} P_{1} \delta_{\text {sd }}^{2}$ and $C=b_{\mathrm{psk}} P_{2} \delta_{\text {rd }}^{2}$.

When a probability density function (pdf) of $w$ is known, we can find an average SER upper bound of the system as follows:

$$
P_{\mathrm{psk}} \leq \int_{0}^{\infty}\left(P_{\mathrm{psk}}^{w}\right) \operatorname{pdf}(w) d w
$$

According to the derivation in the Appendix, the pdf of $w$ can be derived, and the upper bound on the average SER of the proposed system can be expressed as follows [17-19]:

$$
\left(P_{\mathrm{psk}}\right) \leq \frac{(M-1)}{M} \times \frac{N_{0}^{2}}{\left(N_{0}+2 A\right)\left(N_{0}+C\right)} \times \frac{\delta_{\mathrm{rd}}^{2}}{\left(\delta_{\mathrm{sr}}^{2}+\delta_{\mathrm{rd}}^{2}\right)}
$$

where $A=b_{\mathrm{psk}} P_{1} \delta_{\mathrm{sd}}^{2}$ and $C=b_{\mathrm{psk}} P_{2} \delta_{\mathrm{rd}}^{2}$.

\subsection{The proposed optimum relay selection technique}

It is well aware that the diversity gain of the cooperative communication system can be increased by the number of the relay in the system [1]. In this section, we proposed the optimum relay selection technique such that the 
source node can select the optimum cooperative relay depending on the channel quality of each link. Assuming that all channel information in the system is known to the source node by the virtue of some feedback channels, the source node is able to exploit this information to find the optimum relay for cooperative communications by using the following rule:

$$
\min \left(P_{\mathrm{psk}}\left(R_{1}\right), P_{\mathrm{psk}}\left(R_{2}\right)\right)
$$

where $R_{1}$ stands for the relay 1 , and $R_{2}$ stands for the relay 2 .

The concept of optimum relay selection can be explained as follows (Figure 3): Referring to the system model in section 2 , the source node could be possibly transmitting the data to the destination by using relay 1 or relay 2. From (32), the source node needs the feedback channel information of source to relay and relay to destination links for calculating the upper bound on averaged SER for relay 1 and relay 2 , respectively. Thus, the optimum relay that provides the lower probability of error for cooperative communications is selected. In the simulation, the proposed optimum relay selection technique outperforms the fixed relay selection technique. This is because of the diversity gain, which depends on the number of relays, as will be shown in the simulation results (section 5).

\subsection{The optimum power allocation}

In the cooperative communication system, the source node transmits the data to the destination node with the transmission power $P_{1}$, and the relay helps the source node to re-transmit the data with transmission power $P_{2}$. Thus, the total transmission power is equal to $P=P_{1}+P_{2}$, and the transmission power ratio is equal to $P_{\mathrm{r}}$, where $P_{\mathrm{r}}=P_{1} / P_{2}$.

In this section, the optimum power allocation is investigated, in which the transmission power $P_{1}$ and $P_{2}$ could be optimally allocated. The transmission power ratio can be increased or decreased depending on the channel quality of the system. The optimum power allocation strategy can be obtained by minimizing the upper bound on the average SER, i.e. (29), as follows:

Substituting $A=b_{\mathrm{psk}} P_{1} \delta_{\mathrm{sd}}^{2}$ and $C=b_{\mathrm{psk}} P_{2} \delta_{\mathrm{rd}}^{2}$ to arrive at

$$
\begin{aligned}
\min _{P_{1}}\left[\frac{(M-1)}{M}\right. & \times \frac{N_{0}^{2}}{\left(N_{0}+2 b_{\mathrm{psk}} P_{1} \delta_{\mathrm{sd}}^{2}\right)\left(N_{0}+b_{\mathrm{psk}} P_{2} \delta_{\mathrm{rd}}^{2}\right)} \\
& \left.\times \frac{\delta_{\mathrm{rd}}^{2}}{\left(\delta_{\mathrm{sr}}^{2}+\delta_{\mathrm{rd}}^{2}\right)}\right], \text { s.t.P }=P_{1}+P_{2},
\end{aligned}
$$

We solve the optimization problem in (34) by using the standard Lagrangian method to arrive at

$$
\begin{aligned}
& G\left(P_{1}, P_{2}\right)=\left[\frac{(M-1)}{M} \times \frac{N_{0}^{2}}{\left(N_{0}+2 b_{\mathrm{psk}} P_{1} \delta_{\mathrm{sd}}^{2}\right)\left(N_{0}+b_{\mathrm{psk}} P_{2} \delta_{\mathrm{rd}}^{2}\right)}\right. \\
&\left.\times \frac{\delta_{\mathrm{rd}}^{2}}{\left(\delta_{\mathrm{sr}}^{2}+\delta_{\mathrm{rd}}^{2}\right)}\right], \text { s.t.P }=P_{1}+P_{2}, \\
& \frac{\partial G\left(P_{1}, P_{2}\right)}{\partial P_{1}}=\frac{(M-1)}{M} \times \frac{\delta_{\mathrm{rd}}^{2}}{\left(\delta_{\mathrm{sr}}^{2}+\delta_{\mathrm{rd}}^{2}\right)} \\
& \times \frac{N_{0}^{2}\left(2 \delta_{\mathrm{sd}}^{2} N_{0}+2 P_{2} \delta_{\mathrm{sd}}^{2} \delta_{\mathrm{rd}}^{2}\right)}{\left(N_{0}^{2}+2 P_{1} \delta_{\mathrm{sd}}^{2} N_{0}+P_{2} \delta_{\mathrm{rd}}^{2} N_{0}+2 P_{1} P_{2} \delta_{\mathrm{sd}}^{2} \delta_{\mathrm{rd}}^{2}\right)^{2}}
\end{aligned}
$$

By taking the partial derivative on (36) with respect to $P_{1}$, and setting the result to 0 , we have

$$
2 \delta_{\text {sd }}^{2} N_{0}+2 P_{2} \delta_{\text {sd }}^{2} \delta_{\text {rd }}^{2}=\delta_{\text {rd }}^{2} N_{0}+2 P_{1} \delta_{\text {sd }}^{2} \delta_{\text {rd }}^{2} N_{0}^{2}
$$

With the power constraint $P=P_{1}+P_{2}$, the optimum transmission power can be obtained as follows:

$$
\begin{aligned}
& P_{1}=\frac{2 \delta_{\mathrm{sd}}^{2} N_{0}-\delta_{\mathrm{rd}}^{2} N_{0}+2 P \delta_{\mathrm{sd}}^{2} \delta_{\mathrm{rd}}^{2}}{4 \delta_{\mathrm{sd}}^{2} \delta_{\mathrm{rd}}^{2}} \\
& P_{2}=P-\frac{2 \delta_{\mathrm{sd}}^{2} N_{0}-\delta_{\mathrm{rd}}^{2} N_{0}+2 P \delta_{\mathrm{sd}}^{2} \delta_{\mathrm{rd}}^{2}}{4 \delta_{\mathrm{sd}}^{2} \delta_{\mathrm{rd}}^{2}}
\end{aligned}
$$

It could be observed that the optimum power allocation expressions in (38) and (39) at high SNR regimes mainly

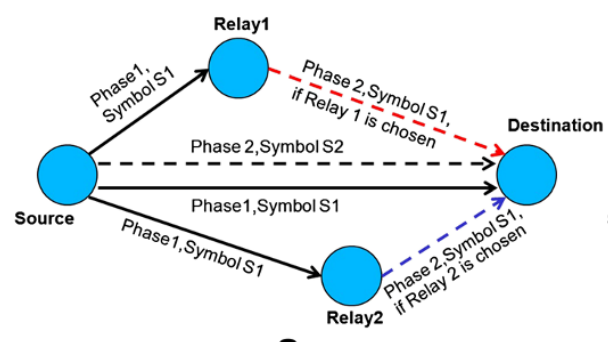

a

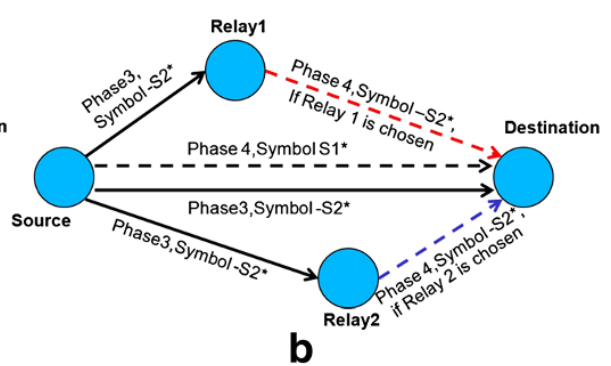

Figure 3 System model of an Alamouti-coded decoded-and-forward protocol with optimum relay selection for cooperative communications in different phases. (a) Phases 1 and 2 and (b) phases 3 and 4. 
depend on the value of noise variances. However, in the low SNR regimes, the transmission power ratio will depend on the channel quality of the source-to-destination and the relay-to-destination links. It could also be observed that if the channel quality of the source-to-destination link is better than the relay-to-destination link, the transmission power ratio $\left(P_{\mathrm{r}}\right)$ will be greater than one. However, if the channel quality of the source-to-destination link is less than the relay-to-destination link, the $P_{\mathrm{r}}$ will be less than one. In the system which has the same channel quality between the source-to-destination link and the relay-to-destination link, the transmission power $P_{1}$ will be equal to $P_{2}$, i.e., $P_{\mathrm{r}}=1$. In addition, the constraint that $P_{1}>0$ and $P_{2}>0$ must be held because the source and relay nodes must be coexisted in order to form the Alamouti-coded cooperative communications.

Therefore, some amount of power must be allocated to both source and relay nodes; as a result, the constraint $P_{1}>0, P_{2}>0$, if and only if $P>0$, will be valid. In other words, if $P_{1}=0$, it is equivalent to the case that there is no source node, and, hence the relay node cannot form the Alamouti-coded cooperative communications with any other node. This is not the considered case in the paper. It is also worth noticing that the power allocation does not depend on the source-to-relay link because the effect of this link has been averaged in the upper bound on the average SER expression in (32), and it results in a multiplicative factor, i.e., $\delta_{\mathrm{rd}}^{2} / \delta_{\mathrm{sr}}^{2}+\delta_{\mathrm{rd}}^{2}$, which is independent of $P_{1}$ and $P_{2}$.

\section{Simulation result}

In this section, based on a computer simulation, a performance evaluation of the proposed Alamouti-coded decode-and-forward protocol with optimum relay selection for cooperative communication will be examined. The simulation is conducted under quasi-static Rayleigh flat fading channel. The digital modulation with QPSK constellation is employed. The total transmit power is fixed to be $P=2 \mathrm{~W}$, and the bandwidth efficiency is 1 $\mathrm{bit} / \mathrm{s} / \mathrm{Hz}$. The variances of channel links will be varied to different values in order to examine the proposed system in various aspects. In simulation results, we define $\left(\delta_{\mathrm{sd}}^{2}, \delta_{\mathrm{sr}}^{2}, \delta_{\mathrm{rd}}^{2}\right)$ as channel variances of the source-todestination, the source-to-relay, and the relay-to-destination links, respectively.

In Figure 4, we present a curve of a simulated average SER versus SNR (dB) for two-user DF cooperative communication systems, including the curve for DF cooperative scheme with the proposed Alamouti's coding. The existing DF cooperative results in higher probability of error than the proposed protocol about 2 to $4 \mathrm{~dB}$.

In Figure 5, we present a curve of a simulated average SER versus SNR (dB) for two users, i.e., one source node and one relay node, in DF cooperative communication systems with C-MRC signal combining, including the curve for a direct transmission scheme, the curve for a DF cooperative scheme, and the curve for an Alamouti-coded decode-and-forward protocol for cooperative communications. It is worth noticing that the proposed cooperative scheme is much superior to the non-cooperative scheme. This is because of the diversity gain achieved in the proposed cooperative communication system. In addition, the different channel variance represents the different channel quality, in which the high value means a good channel because the path loss is small.

In Figure 6, we present a curve of average SER versus SNR (dB) for three-user cooperative communication systems, i.e., one source node and two relay nodes, including the curve for the decode-and-forward protocol with optimum relay selection and Alamouti coding, the proposed Alamouti-coded decode-and-forward protocol with optimum relay selection and an ordinary cooperative decode-and-forward protocol. It is worth noticing that the best relay selection technique is much superior than the existing cooperative protocol. This is because of the diversity gain achieved in the increasing bandwidth efficiency depending on the number of relays. The existing DF cooperative protocol results in higher probability of error than the proposed system over $5 \mathrm{~dB}$ at SER lower than $10^{-3}$. The results show that the Alamouti coding yields the lower probability of error than the existing DF protocol about $1.5 \mathrm{~dB}$ at SNR higher than $20 \mathrm{~dB}$.

In Figure 7, we present the SER of the Alamouti-coded decode-and-forward protocol with optimum relay selection cooperative communications with the different transmission power level $P_{1}$. Note that we define the $\mathrm{SNR}$ as $\mathrm{SNR}=10 \log \left[P / N_{0}\right]$, where $P$ stands for the total transmit power of the system and $N_{0}$ stands for the noise variances of all relay and destination nodes, in which they are assumed identical. It is worth noticing that the power allocation expression in (38) and (39) depend on the system noise variance, i.e. $N_{0}$; the channel variances, i.e. $\delta_{\text {sd }}^{2}$ and $\delta_{\text {rd; }}^{2}$ and the total transmit power $P$. In Figure $7 \mathrm{a}$, we considered the system with all channel variance equals to 1 . From this figure, we can see that the ratio $P_{1} / P=0.5$ provides the best performance for the difference SNR levels $(10,20$, and $30 \mathrm{~dB})$. In a high SNR regime, the optimum power allocation results in an equal power allocation as shown in Figure 7b, where variance $(0.1,1,1)$ is considered. In a low SNR regime, the power allocation results in two cases: (A) small channel variance in the source-to-destination link compared with the relay-to-destination link, where variance $(0.1,1,1)$ and (B) high channel variance in the source-todestination link compared with the relay-to-destination link, where variance $(1,1,0.1)$. For case A in Figure $7 \mathrm{c}$, we can observe that less power will be allocated to the source 


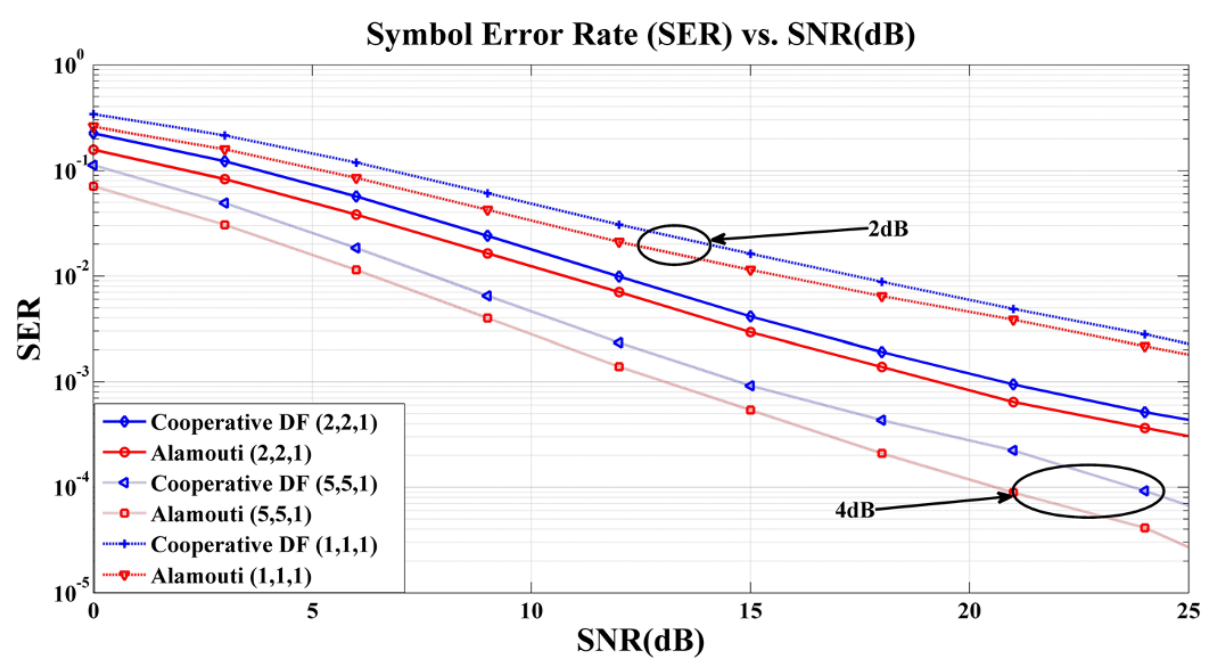

Figure 4 Simulated SER curves of decode-and-forward protocol vs. Alamouti-coded decode-and-forward protocol for two-user cooperative communications.

node and more power will be allocated to the relay node. This is because the SNR in the source-todestination link in (23) and (24) contains a factor of $(1+w)$ which is higher than factor $(w)$ in the relay-todestination link. Therefore, more power will be allocated to the relay node in order to maximize the total SNR. For case B in Figure 7c, we can also observe that more power will be allocated to the source node and less power will be allocated to the relay node. In this case, the C-MRC weight $w$ approaches 1 , which is the maximum value. Hence, at low SNR with the small channel variances in the relay-to-destination link, the proposed system will perform close to the non-cooperative communication system in order to minimize the effect of uncertainty in the relay link.

In Figure 8, the optimum power allocation of the proposed system is presented. The curves show the comparison between theoretical upper bound on error probability of the proposed protocol with and without the optimum power allocation techniques. In the system, which has the high channel quality (i.e., channel variance of 5 for all of channel links) and the low channel quality system (i.e., channel variance of 0.5 for all of channel links), the results confirm that the proposed protocol yields the same error probability as the equal power allocation because the transmission power $P_{1}$ and $P_{2}$ are almost identical.

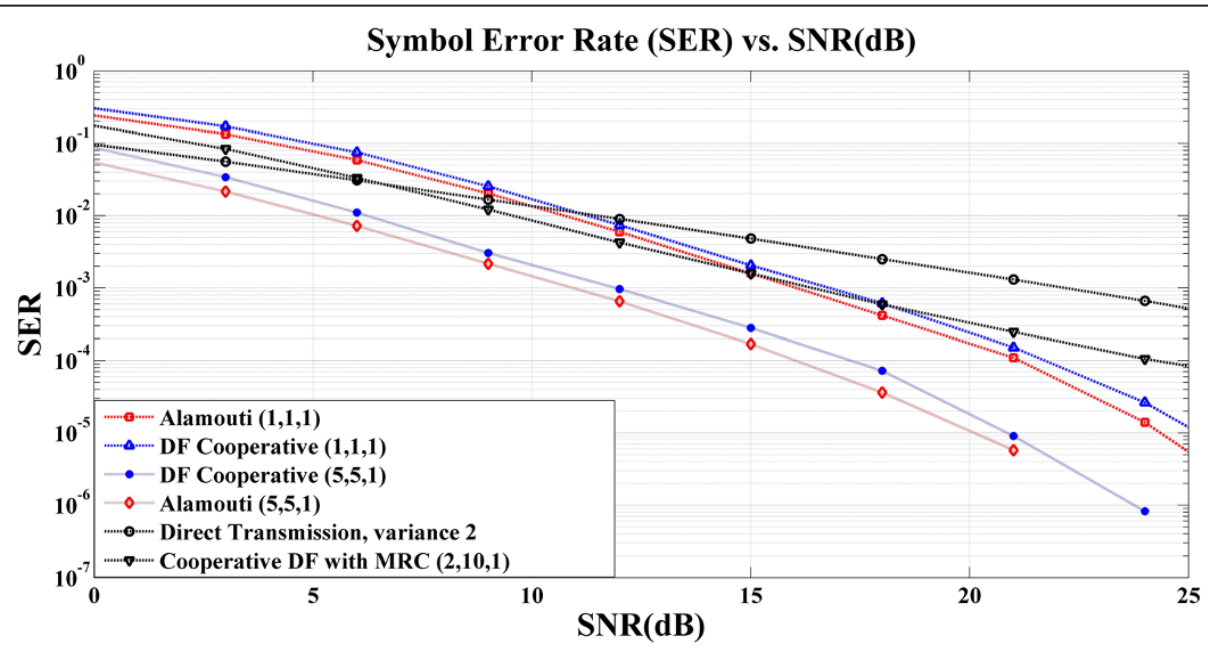

Figure 5 Simulated SER curves of decode-and-forward protocol vs. Alamouti-coded decode-and-forward protocols for two-user cooperative communications with C-MRC signal combining. 


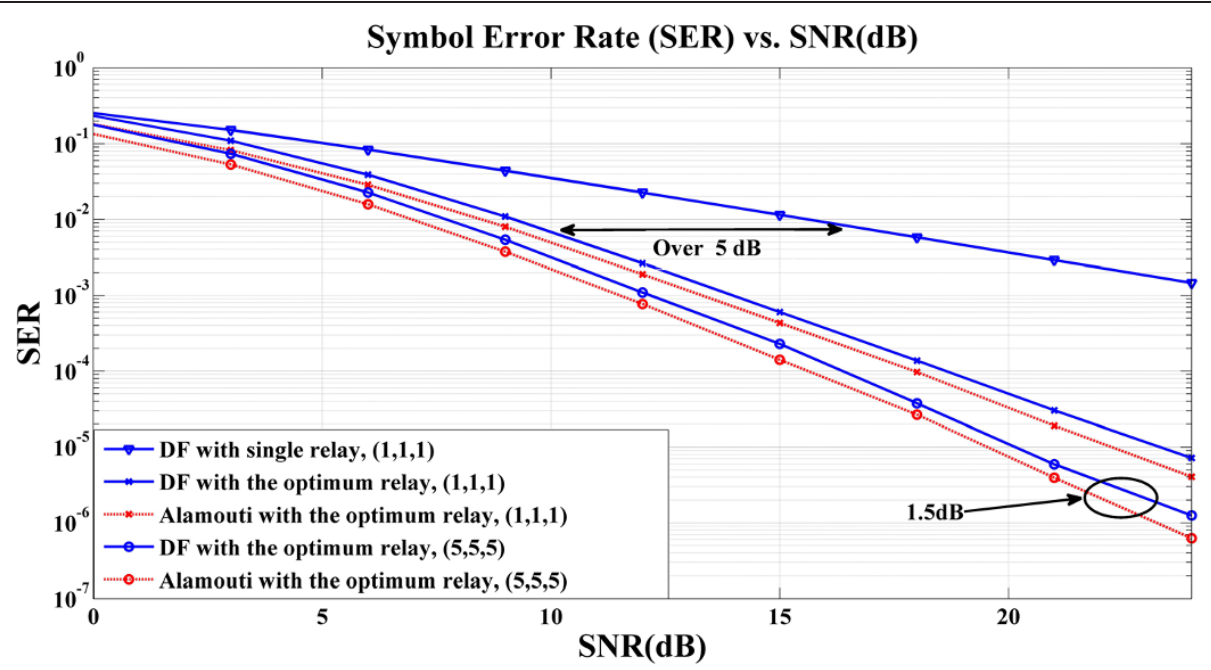

Figure 6 Simulated SER curves of decode-and-forward protocol vs. Alamouti-coded decode-and-forward protocol with optimum relay selection for three-user cooperative communications.

However, in the case that the channel variance is equal to 0.05 for the source-to-destination link, and the channel variance of 5 for the source-to-relay link and the relay-to-destination link, the system which employs the optimum power allocation technique shows the lower error probability at low SNR regimes. At high SNR regimes, the transmission power resulted from the optimum power allocation is the same as the equal power allocation because the noise variance in (38) and (39) have an insignificant influence.

In Figure 9, we present the comparison between simulated SER and the theoretical upper bound on an average SER of the proposed system with and without the optimum power allocation technique. These results show

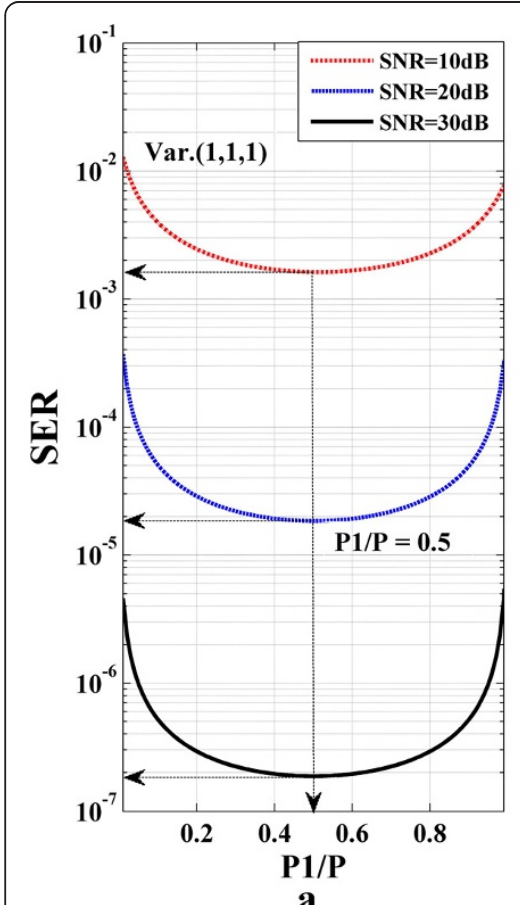

a

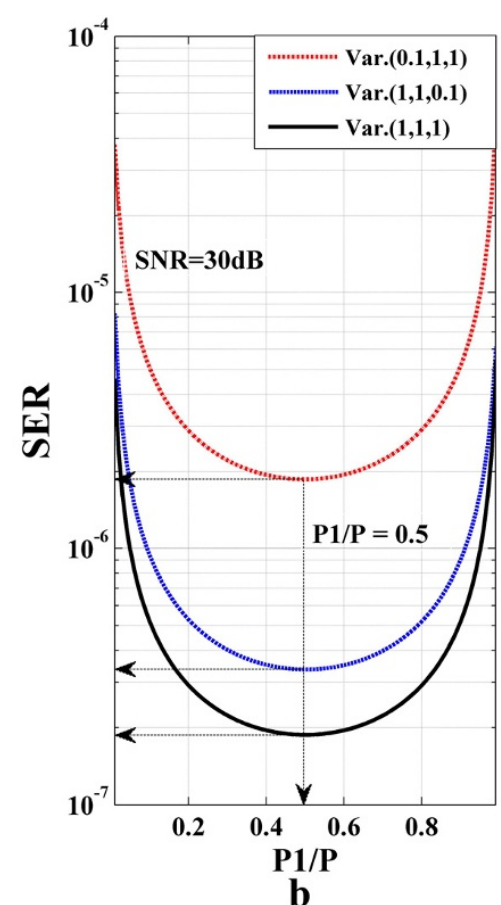

b

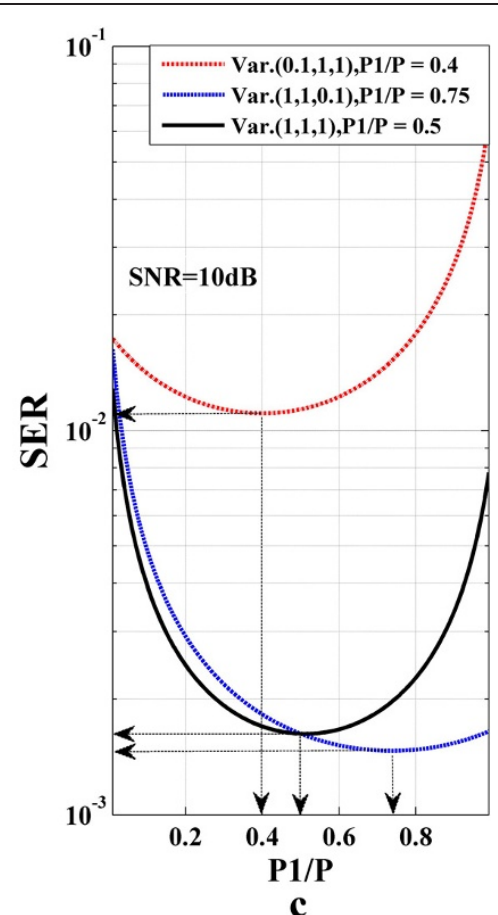

c

Figure 7 Simulated SER of Alamouti-coded decode-and-forward protocol with optimum relay selection cooperative communications. Different transmission power levels: $P_{1}$ and $P_{2}$. (a) Fixed channel variances with different SNR values. (b) High SNR regime with different channel variances. (c) Low SNR regime with different channel variances. 


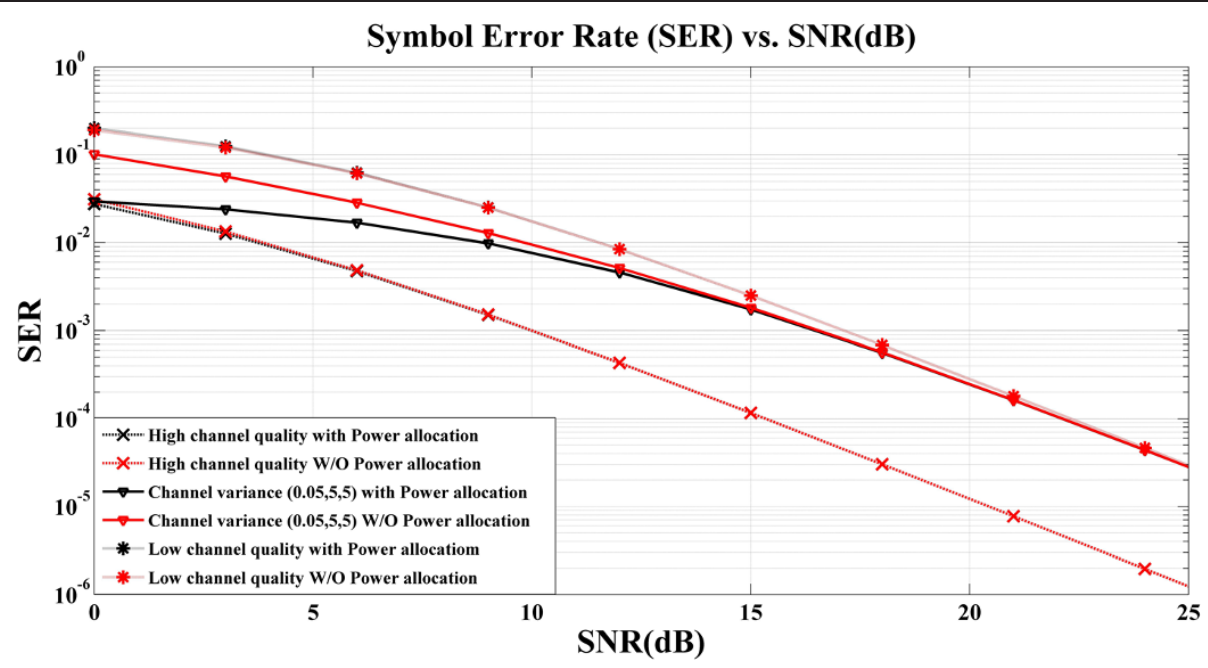

Figure 8 Theoretical error probability curves with and without an optimum power allocation.

that theoretical curves perform close to the simulation curves in all case. In addition, in the case of optimum power allocation, the proposed system yields a lower probability of error in low SNR regimes, and the probability of error is close to the equal power allocation case in high SNR regimes.

\section{Conclusion}

In this paper, we have proposed the Alamouti-coded decode-and-forward protocol with optimum relay selection for three-user cooperative communications. The optimum relay selection protocol in the scenario of a high channel link, i.e., a channel variance of the sourceto-destination link, the source-to-relay link, and the relay-to-destination link are equal to 5 , significantly outperforms the existing decode-and-forward (DF) protocol, where the SNR improvement over $5 \mathrm{~dB}$ at SER lower than $10^{-3}$ is observed. Furthermore, the proposed Alamouti's coding scheme performs better than existing DF protocol of about $1 \mathrm{~dB}$ at all SER regimes. In the performance analysis, the derived probability of error shows a close result to the simulated one. In addition, the derived upper bound on an average SER could be used to determine the optimum transmission power for the source node and the relay node. It is worth noticing that the optimum power allocation could enhance the error probability of the system effectively, especially in the low SNR regimes. Furthermore, in the case of no information about the channel variances, the equal power allocation could be fairly used instead as confirmed by the simulation results in high SNR regimes. Furthermore, the proposed protocol achieves a full diversity gain by the virtue of increasing a number of signal transmissions in the relaying phases.

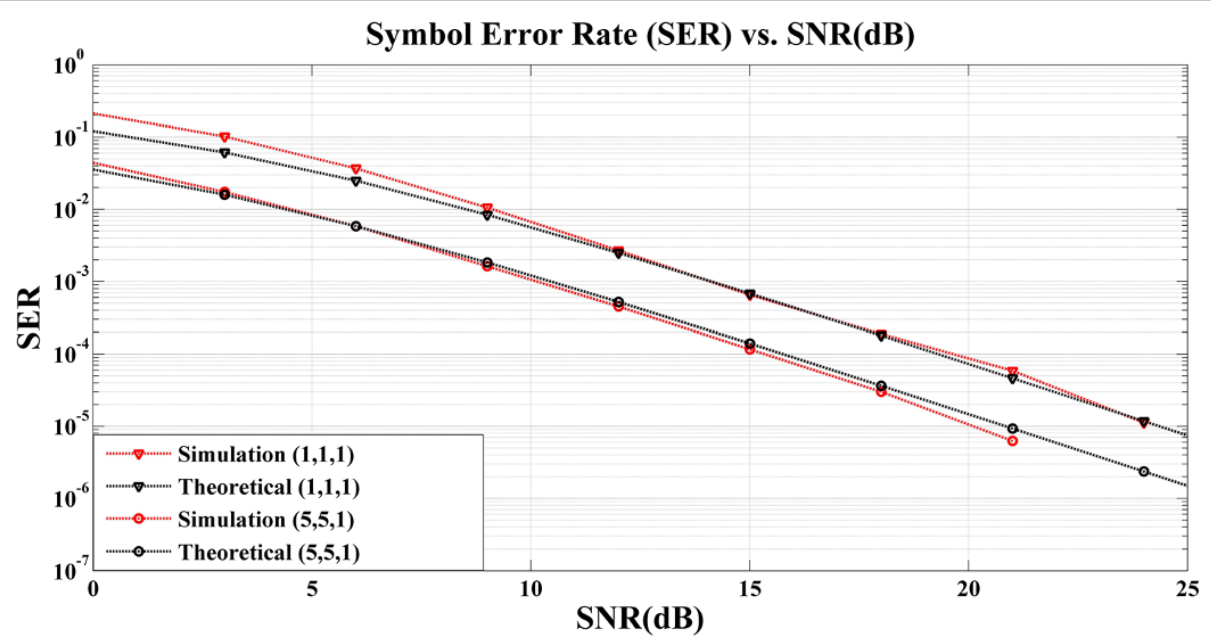

Figure 9 A curve of the simulated error probability vs. the theoretical upper bound on an average SER. 


\section{Appendix}

The probability density function (pdf) of $w$ can be derived, where $w$ is the weighting function of the C-MRC signal combining technique, as follows,

$$
\operatorname{pdf}(w)=\operatorname{pdf}\left(\frac{\min (X, Y)}{Y}\right)
$$

Suppose that $X$ and $Y$ are independent exponential random variables, with densities $a e^{-a u}$ and $b e^{-b u}, u>0$, respectively. For any $0<x<1$ and $s>0, a$ is the channel variance between source to relay link, and $b$ is the channel variance between relay to destination link.

$$
P(\min (X, s) \leq x s)=P(X \leq x s)=1-e^{-a x s}
$$

For any $0<\mathrm{x}<1$, by the law of total probability (conditioned on $Y$ )

$$
\begin{aligned}
& P\left(\frac{\min (X, Y)}{Y} \leq x\right)=\int_{0}^{\infty} \\
& P\left(\frac{\min (X, s)}{s} \leq x\right) b e^{-b s} \\
& d s=\int_{0}^{\infty} P(\min (X, s) \leq x s) b e^{-b s} d s
\end{aligned}
$$

Hence,

$$
\begin{aligned}
& P\left(\frac{\min (X, Y)}{Y} \leq x\right)=\int_{0}^{\infty}\left(1-e^{-a x s}\right) b e^{-b s} \\
& d s=1-b \int_{0}^{\infty} e^{-(a x+b) s} \\
& d s=1-\frac{b}{a x+b}
\end{aligned}
$$

The probability density function is obtained by the derivative of (43). The ratio, called $R(R=\min (X, Y) / Y)$, has positive mass at $x=1$; therefore, the density of $\mathrm{R}$ on $(0,1)$ does not integrate to 1 . Specifically, the probability density function $f_{R}$ of $\mathrm{R}$ on $(0,1)$ is given by

$$
f_{R}(x)=\frac{d}{d x}\left(1-\frac{b}{a x+b}\right)=\frac{a b}{(a x+b)^{2}}, 0<x<1
$$

It holds

$$
\int_{0}^{1} f_{R}(x) d x=\left.\left(1-\frac{b}{a x+b}\right)\right|_{0} ^{1}=1-\frac{b}{a+b}
$$

which is less than 1 . This implies that $P(R=1)=b /(a+b)$. Indeed, $P(R=1)=P(\min (X, Y)=Y) P(Y \leq X)$, and hence, by the law of total probability (conditioning on $X$ ),

$$
\begin{aligned}
P(R=1) & =\int_{0}^{\infty} P(Y \leq u) a e^{-a u} d u F_{R}(x)=1-\frac{b}{a x+b}, 0<x<1 \\
& =1-\frac{a}{a+b}=\frac{b}{a+b}
\end{aligned}
$$

In summary, the ratio $R$ is a random variable supported on $[0,1]$. It has a distribution function $F_{R}$, given by

$$
=\int_{0}^{\infty}\left(1-e^{-b u}\right) a e^{-a u} d u=1-a \int_{0}^{\infty} e^{-(a+b) u} d u
$$

and $F_{R}(1)=1$.

Thus, $F_{R}$ has jump discontinuity at $x=1$, where

$$
F_{R}(1)-\lim _{x \rightarrow 1^{-}} F_{R}(x)=1-\lim _{x \rightarrow 1^{-}}\left(1-\frac{b}{a x+b}\right)=\frac{b}{a+b}
$$

which is the probability $P(R=1)$. In particular, the density function of $R$ exists only for $x<1$. It is given by

$$
f_{R}(x)=\frac{a b}{(a x+b)^{2}}, 0<x<1
$$

and

$$
F_{R}(x)=1-\frac{b}{a x+b}, 0<x<1
$$

Hence, given $a$ being the variance of a random variable $x$ and $b$ being the variance of a random variable $y$, the pdf of $R=\min (X, Y) / Y$ could be expressed as

$$
f_{R}(x)=\frac{a b}{(a x+b)^{2}}+\frac{b}{a+b} \delta(x-1)
$$

Substituting (51) into (31), we have an upper bound on the average SER as follows:

$$
\begin{aligned}
\left(P_{\mathrm{psk}}\right) \leq \frac{(M-1)}{M} & \times \int_{0}^{1} \frac{N_{0}^{2}}{\left(N_{0}+(1+w) A\right)\left(N_{0}+w C\right)} \times \frac{a b}{(a w+b)^{2}} d w \\
& +\frac{(M-1)}{M} \int_{1}^{1} \frac{N_{0}^{2}}{\left(N_{0}+(1+w) A\right)\left(N_{0}+w C\right)} \\
& \times \frac{b}{(a+b)} \delta(w-1) d w
\end{aligned}
$$

By using a partial fraction expansion technique, we can prove that

$$
\begin{aligned}
\frac{N_{0}^{2}}{\left(N_{0}+(1+w) A\right)\left(N_{0}+w C\right)} & \times \frac{a b}{(a w+b)^{2}}=\frac{X a}{(N o+A+A w)} \\
& +\frac{X b}{(N o+C w)}+\frac{X c}{(a w+b)^{2}}+\frac{X d}{(a w+b)}
\end{aligned}
$$


Hence,

$$
\begin{aligned}
\left(P_{\mathrm{psk}}\right) \leq & \frac{(M-1)}{M} \frac{X a}{A} \ln \left(\frac{N_{0}+2 A}{N_{0}+A}\right)+\frac{X b}{C} \ln \left(\frac{N_{0}+C}{N_{0}}\right) \\
& -X c\left[\frac{1}{a(a+b)}+\frac{1}{a b}\right]+\frac{X d}{a} \ln \left(\frac{a+b}{b}\right) \\
& +\frac{N_{0}^{2}}{\left(N_{0}+2 A\right)\left(N_{0}+C\right)} \times \frac{b}{(a+b)} \\
& -X c\left[\frac{1}{a(a+b)}+\frac{1}{a b}\right]+\frac{X d}{a} \ln \left(\frac{a+b}{b}\right) \\
& +\frac{N_{0}^{2}}{\left(N_{0}+2 A\right)\left(N_{0}+C\right)} \times \frac{b}{(a+b)}
\end{aligned}
$$

where $X a=\frac{N_{0}^{2} a b}{\left(N_{0}+\left(-\frac{N_{0}}{A}-1\right)\right)\left(\left(-\frac{N_{0}}{A}-1\right) a+b\right)^{2}}, X b=\frac{N_{0}^{2} a b}{\left(N_{0}+\left(1-\frac{10}{C}\right) A\right)\left(-\frac{N_{0}}{C} a+b\right)^{2}}, \quad X c=$ $\frac{N_{0}^{2} a b}{\left(N_{0}+\left(1-\frac{b}{a}\right) A\right)\left(N_{0}-\frac{b}{a} C\right)}$, and $X d=\frac{N_{0}^{2} a b-X a N_{0} b^{2}-X b\left(N_{0}+A\right) b^{2}-X c\left(N_{0}^{2}+N_{0} A\right)}{\left(N_{0}^{2}+N_{0} A\right) b}$.

It could be verified that $X a, X b, X c$, and $X d$ are invalid values of this expansion because these parameters will make the total probability of error less than zero, i.e., $P_{\mathrm{psk}}<0$. Therefore, we can reasonably ignore them. Finally, the total probability of error of the system can be shown as stated in equation (32)

$$
\left(P_{\mathrm{psk}}\right) \leq \frac{(M-1)}{M} \times \frac{N_{0}^{2}}{\left(N_{0}+2 A\right)\left(N_{0}+C\right)} \times \frac{\delta_{r d}^{2}}{\left(\delta_{s r}^{2}+\delta_{r d}^{2}\right)}
$$

\section{Competing interests}

The authors declare that they have no competing interests.

\section{Acknowledgements}

This work is financially supported by the Thailand Research Fund (TRF) and the Office of the Higher Education Commission (OHEC) under the grant number MRG5080388.

\section{Author details}

${ }^{1}$ The Sirindhorn International Thai-German Graduate School of Engineering (TGGS), King Mongkut's University of Technology North Bangkok, Bangkok 10800, Thailand. ${ }^{2}$ Department of Electrical Engineering, Chulalongkorn University, Bangkok 10330, Thailand. Institute for Communication Technologies and Embedded Systems (ICE), RWTH Aachen University, 52062 Aachen, Germany.

Received: 30 November 2012 Accepted: 11 May 2014

Published: 9 July 2014

\section{References}

1. JN Laneman, DNC Tse, GW Wornell, Cooperative diversity in wireless networks: efficient protocols and outage behavior. IEEE Trans Inform Theor 50, 3062-3080 (2004)

2. SM Alamouti, A simple transmit diversity technique for wireless communications. IEEE J Sel Area Comm 16, 1451-1458 (1998)

3. S Weifeng, A Sadek, AK Liu, SER performance analysis and optimum power allocation for decode-and-forward cooperation protocol in wireless networks, in Paper presented at the IEEE Conference on Wireless Communication and Networking, New Orleans, 13-17 March 2005

4. TWang, A Cano, GB Giannakis, JN Laneman, High-performance cooperative demodulation with decode-and-forward relays. IEEE Trans Wireless Comm 55(No. 7), 1427-1438 (2007)

5. Y Hua, Y Mei, Y Chang, Wireless antennas - making wireless communications perform like wireless communications, in IEEE Topical
Conference on Wireless Communication Technology, Honolulu pp. 47-73. 15-17 Oct 2003

6. TQ Duong, H Dac-Binh, T Hoai-An, V Nguyen-Son, Symbol error probability of Distributed-Alamouti scheme in wireless relay networks, in IEEE Vehicular Technology Conference, VTC spring, 2008, Singapore, pp. 648-652. 11-14 May 2008

7. Y Chang, Y Hua, Diversity analysis of orthogonal space-time modulation for distributed wireless relays, in Proceedings of the IEEE International Conference on Acoustics, Speech, and Signal Processing (ICASSP '04), Montreal, Canada, 2004, pp. 561-564. 17-21 May

8. S Atapattu, N Rajatheva, Analysis of Alamouti code transmission over TDMA-based cooperative protocol, in Paper presented at the IEEE Vehicular Technology Conference, 2008, VTC Spring 2008, Singapore, 11-14 May 2008

9. A Bletsas, H Shin, MZ Win, A Lippman, A simple cooperative diversity method based on network path selection. IEEE Journal on Selected Areas in Communications 24(3), 659-672 (2006)

10. SS Ikki, MH Ahmed, Performance analysis of adaptive decode-and-forward cooperative diversity network with best-relay selection. IEEE Transactions on Communications 58(1), 68-72 (2010)

11. A Adinoyi, Y Fan, H Yanikomeroglu, HV Poor, F Alshaalan, Performance of selection relaying and cooperative diversity. IEEE Trans Wireless Comm 8(12), 5790-5795 (2009)

12. X Jin, JS No, DJ Shin, Relay selection for decode-and-forward cooperative network with multiple antennas. IEEE Trans Wireless Comm 10(12), 4068-4079 (2011)

13. DG Brennan, Linear diversity combining techniques. Proc IEEE 91(2), 331-356 (2003)

14. W Swasdio, C Pirak, A novel Alamouti-coded decode-and-forward protocol for cooperative communications, in IEEE Conference, (TENCON, Fukuoka, 2010), pp. 2091-2095. 21-24 Nov 2010

15. W Swasdio, C Pirak, G Ascheid, Alamoti-coded decode-and-forward protocol with optimum relay selection for cooperative communications, in International Congress on Ultra Modern Telecommunications and Control Systems and Workshops (ICUMIT), Budapest, 5-7 Oct 2011

16. S Weifeng, A Sadek, AK Liu, Cooperative Communication and Networking (Cambridge University Press, Cambridge, 2009)

17. MO Hasna, MS Alouini, End-to-end performance of transmission systems with relays over Rayleigh-fading channels. IEEE Trans Wireless Comm 2, 1126-1131 (2003)

18. MK Simon, MS Alouini, Digital Communication over Fading with Formulas, Graphs and Mathematical Tables, 9th edn. (Dover, New York, 1970)

19. A Papoulis, Probability, Random Variables, and Stochastic Processes (McGraw-Hill, New York, 1991)

doi:10.1186/1687-1499-2014-112

Cite this article as: Swasdio et al:: Alamouti-coded decode-and-forward protocol with optimum relay selection and power allocation for cooperative communications. EURASIP Journal on Wireless Communications and Networking 2014 2014:112.

\section{Submit your manuscript to a SpringerOpen ${ }^{\odot}$ journal and benefit from:}

- Convenient online submission

- Rigorous peer review

- Immediate publication on acceptance

- Open access: articles freely available online

- High visibility within the field

- Retaining the copyright to your article

Submit your next manuscript at $\gg$ springeropen.com 